\title{
Effect of Refined Nutrition Management on Nutritional Status of Patients with Decompensated Liver Cirrhosis
}

\author{
Weiying Li, Yusi Li, Min Liu, Liangqing Gao, Yujing Zhou* \\ Department of Digestive, The Fifth Affiliated Hospital of Sun Yat-Sen University, Zhuhai 519000, China. E-mail: \\ zg18836@163.com
}

\begin{abstract}
Objective: To analyze the effect of refined nutrition management on nutritional status of patients with decompensated liver cirrhosis. Methods: 100 cases of patients with decompensated liver cirrhosis treated in the author's hospital from August 2018 to December 2019 were selected. The patients were divided into the control group and the observation group randomly and they were given routine nursing management and refined nutrition management respectively. The nutritional status of the patients in the two groups was compared. Results: The post-intervention level of albumin, hemoglobin and prealbumin in the observation group was significantly higher than those in the control group after intervention, while total bilirubin was significantly lower than that in the control group, $\mathrm{p}<0.05$. Conclusion: The application of refined nutrition management in patients with decompensated liver cirrhosis can promote the improvement of nutritional status of the body, which has the value of further promotion and implementation.
\end{abstract}

Keywords: Decompensated Liver Cirrhosis; Refined Nutrition Management; Nutritional Status

In recent years, abnormal metabolism and malnutrition have gradually become outstanding problems in changes of people's eating habits and living environment, and the clinical incidence rate of liver cirrhosis is increasing year by year ${ }^{[1]}$. Liver is an important organ for nutrition metabolism. Patients with liver cirrhosis have malnutrition and metabolic disorders to varying degrees, of whom nutritional imbalance is a typical manifestation. Relevant clinical statistics showed that it is of great significance to implement scientific and reasonable nursing management for patients with decompensated liver cirrhosis in improving their survival rate and quality of life. This paper mainly analyzes the effect of refined nutrition management on nutritional status of patients with decompensated liver cirrhosis, aiming to provide reference for the selection of clinical nursing programs in the future. The specific research contents are summarized as follows.

\section{Materials and methods}

\subsection{Materials}

100 cases of patients with decompensated liver cirrhosis treated in the author's hospital were selected as the observation objects of this paper, and the admission time was from August 2018 to December 2019. The patients were divided into the control group and the observation group randomly with 50 patients in each group. This study was approved by the Hospital Ethics Committee and it got the informed content of all patients.

The control group: according to the sex of the patients, the numbers of male cases and female cases

\footnotetext{
Copyright (C) 2020 Weiying Li et al.

doi: $10.18686 /$ jn.v9i2.168

This is an open-access article distributed under the terms of the Creative Commons Attribution Non-Commercial License

(http://creativecommons.org/licenses/by-nc/4.0/), which permits unrestricted non-commercial use, distribution, and reproduction in any medium, provided the original work is properly cited.
} 
were 29 and 21 respectively; the average age was (52.73 $\pm 4.96)$ years old; the average course of the disease was $(1.95 \pm 0.24)$ years.

The observation group: according to the sex of the patients, the numbers of male cases and female cases were 31 and 19 respectively; the average age was (52.68 \pm 4.85 ) years old; the average course of the disease was $(1.79 \pm 0.16)$ years.

The data of the patients with decompensated liver cirrhosis in the two groups was compared, $\mathrm{p}>0.05$.

Selection criteria: patients diagnosed with decompensated liver cirrhosis by clinical examination; patients with normal communication and cognitive abilities; patients with complete clinical medical records.

Exclusion criteria: patients with severe cardiac and pulmonary dysfunction; patients with diabetic nephropathy; patients with consciousness disorder that may affect the nursing work; patients with a history of mental disease; patients who lost contact.

\subsection{Methods}

Patients in the control group were treated with routine nursing. Based on the understanding ability of the patients, centralized explanation was carried out in the form of consultation and lectures to explain the effect of protein on the human body and to introduce the specific causes of hypoproteinemia in decompensated liver cirrhosis to the patients. The patients were told to increase the intake of foods rich in vitamins and high-quality protein in their diet and to develop good living habits and eating habits. Doctors carried out effective and active communication with patients. When patiently listening to patients' ideas, they mastered the factors affecting patients' psychology and emotions, so as to improve their mood and decrease negative emotions. Moreover, patients were told to rest in bed in a quiet and comfortable environment. Nursing work should be carried out other than their rest time to avoid affecting their rest.

The patients in the observation group were given refined nutrition management, the details of which are as follows.

(1) Most patients with decompensated liver cirrhosis suffer from portal hypertension and esophageal and gastric varices to varying degrees. If they eat hard and coarse food, it is easy to cause phleborrhexis that increases bleeding rate. In addition, as the pressure in varicose veins increases, it is very likely to induce massive hemorrhage of upper gastrointestinal tract if phleborrhexis occurs, and it may even threaten the life in serious cases. Therefore, patients in such condition need to take soft food for a long time and they are forbidden to take hard, coarse and excitant food.

(2) If patients have no hemorrhage in upper gastrointestinal trace or hepatic encephalopathy, their diet is mainly bland digestible food with high protein, high vitamin, low salt, and low fat. Such patients should also appropriately increase the intake of various vitamins, folic acid and pantothenic acid, as well as the intake of fresh fruits and vegetables under the eating principle of small amount and multiple meals. They take in about $11700 \mathrm{KJ}$ of calories, $120 \mathrm{~g}$ of protein and $50 \mathrm{~g}$ of fat per day. As for patients with serious damage in the liver function, they should reduce the intake of protein in the diet, and after the recovery of the disease, they increase it appropriately. The daily protein intake is usually below $50 \mathrm{~g}$, which is mainly vegetable protein. If there is bleeding symptoms associated, fasting intervention is required. After bleeding disappears, the diet will gradually change from complete liquid diet to low-fat semi-liquid diet and soft food.

(3) If the patient has symptoms of ascites and edema of both lower limbs, the daily intake of water should be strictly limited. The patient should be told to reduce the amount of drinking water as much as possible unless thirsty. The daily amount of drinking water should be $<$ $1000 \mathrm{ml}$.

\subsection{Observation indicators}

The nutritional status (albumin, hemoglobin, prealbumin and total bilirubin) of two groups of patients with decompensated liver cirrhosis were observed and recorded.

\subsection{Statistical treatment}

SPSS20.0 statistical software was used to process the relevant index and data of the two groups of patients with decompensated liver cirrhosis in this study. The mean \pm standard deviation refers to the index of nutritional status, assessed by t-test. $\mathrm{P}<0.05$ indicates that there is a statistical significance in the difference between the data. 


\section{Experimental results}

As shown in Table 1, the level of albumin, hemoglobin and prealbumin in the observation group were significantly higher than those in the control group, while the total bilirubin was significantly lower than that in the control group, $\mathrm{p}<0.05$.

\begin{tabular}{lllll}
\hline Group & Albumin $(\mathbf{g} / \mathbf{L})$ & Hemoglobin $(\mathbf{g} / \mathbf{L})$ & Prealbumin $(\mathbf{m g} / \mathbf{L})$ & $\begin{array}{l}\text { Total } \\
(\text { umol/L) }\end{array}$ \\
\hline Control group & $28.85 \pm 2.76$ & $97.92 \pm 4.35$ & $135.92 \pm 5.68$ & $70.36 \pm 1.12$ \\
Observation & $34.93 \pm 3.04$ & $115.75 \pm 5.94$ & $168.03 \pm 7.17$ & $50.98 \pm 0.89$ \\
group & & & \\
t & 10.47 & 17.12 & 24.82 & 95.79 \\
$\mathrm{P}$ & 0.01 & 0.01 & 0.01 & 0.01 \\
\hline
\end{tabular}

Table 1. Comparison of nutritional status of two groups' patients with decompensated liver cirrhosis $(n=50)$

\section{Discussion}

Liver cirrhosis is one of the liver diseases with high incidence in clinic at present. It has main characteristics of many complications, long treatment period and poor prognosis $^{[2]}$. Since liver is an organ for human body to carry out nutrient metabolism, patients with decompensated liver cirrhosis suffer from various degrees of nutritional metabolic disorders, which lead to increased risks of ascites, hypoproteinemia, anemia and water-sodium retention. Therefore, clinical medical personnel need to implement targeted nutritional management according to the patient's condition and the body's nutritional status so as to improve the body's nutritional status ${ }^{[3,4]}$.

Routine nursing only pays attention to the basic nursing intervention of patients, which has mechanical and one-sided defects, and it cannot achieve ideal effects in improving the nutritional status of the body ${ }^{[5]}$. Refined nutrition management means that medical staff should guide patients to increase the intake of food with protein, vitamins and carbohydrate in their diet according to the patient's condition and the body's tolerance, in order to maintain the balance and sufficiency of nutrition in daily $\operatorname{diet}^{[6]}$. Through reasonable diet, the risk of bleeding can be reduced. If appropriate, some patients should increase protein intake in their diet to improve the body condition in strict control of intaking water-electrolyte to relieve the degree of edema in lower limbs and $\operatorname{ascites}^{[7,8]}$.

In this study, the nutritional status of patients in the observation group are significantly better than that in the control group, which confirms that refined nutrition management can improve nutritional status of patients with decompensated liver cirrhosis. The reason may be that during the implementation of refined nutrition management, nursing staff can make a diet plan according to the patient's diet habit, nutrition composition in food, disease condition, etc., thus ensuring the intake of nutrients required by the patient's body in the diet, and staff reasonably adjust the patient's diet structure, so as to reduce the risk of bleeding and to relieve the symptoms of edema in lower limbs and ascites ${ }^{[9,10]}$.

To sum up, the application of refined nutrition management in patients with decompensated liver cirrhosis can improve their nutritional status, which has the value of further promotion and application in clinical practice in the future.

\section{References}

1. Zhang X, Deng J, Zhang $\mathrm{N}$, et al. Effect of nutritional risk screening combined with individualized nutrition program on patients with decompensated hepatocirrhosis. Shanghai Nursing 2019; 19(4): 20-23. doi: 10.3969/j.issn.1009-8399.2019.04.004.

2. Wang N, Zhuang Y, Liao W, et al. Effects of oral nutritional supplements on the biochemical indexes and outcomes of patients with decompensated liver cirrhosis and malnutrition. Journal of Navy Medicine 2018; 39(6): 563-567. doi: 10.3969/j.issn.1009-0754.2018.06.029.

3. Song J, Li H, Qi X, et al. The relationship between nutritional status and prognosis of patients with decompensated hepatitis B virus-related liver cirrhosis. Parenteral \& Enteral Nutrition 2018; 25(6): 32-34+40. doi: 10.16151/j.1007-810x.2018.11.006.

4. Zhang X, Deng J, Zhang N, et al. Intervention study on individualized protein-calorie diet in patients 
with decompensated hepatitis B cirrhosis. Chinese Nursing Research 2019; 33(9): 1613-1615. doi: 10.12102/j.issn.1009-6493.2019.09.036.

5. Chen X, Zheng X, Peng C. Clinical etiology and characteristics of peripheral blood and myelogram in patients with decompensated liver cirrhosis (in Chinese). Chinese Journal of Health Laboratory Technology 2019; 29(12): 1522-1524.

6. Huang H, Qian X, Chen X, et al. Application of empowerment education concept in the health education for decompensated patients with hepatitis B cirrhosis. Chinese Journal of Modern Nursing 2018; 24(29): 3527-3531. doi: 10.3760/cma.j.issn.1674-2907.2018.29.012.

7. Chang C, Wang C. Application and effect of nursing health prescription combined with targeted nursing intervention ("innovative nursing intervention") in patients with decompensated hepatitis B cirrhosis (in Chinese). China Health Care and Nutrition 2018; 28(11): 47-48.

8. $\mathrm{Xu} \mathrm{F}, \mathrm{Wu} \mathrm{H}$. Effect of individualized disease management intervention on the medication beliefs and compliance among patients with decompensated cirrhosis. Chinese Journal of Modern Nursing 2018; 24(12): 1457-1460. doi: 10.3760/cma.j.issn.1674-2907.2018.12.024.

9. Qi Y. Effect of psychological nursing on mental health of patients with decompensated cirrhosis (in Chinese). Clinical Research and Practice 2018; 3(19): 165-166.

10. Song X, Liu X, Chen N, et al. Application effect of pathway-based health education combined with psychological nursing in patients with decompensated cirrhosis (in Chinese). Modern Digestion \& Intervention 2018; 23(3): 408-410. doi: 10.3969/j.issn.1672-2159.2018.03.051. 